\title{
Hidatidosis humana en menores de edad: manifestación de fracaso en las medidas de control y prevención. Chile, 2001-2011
}

\author{
Paulina Martínez
}

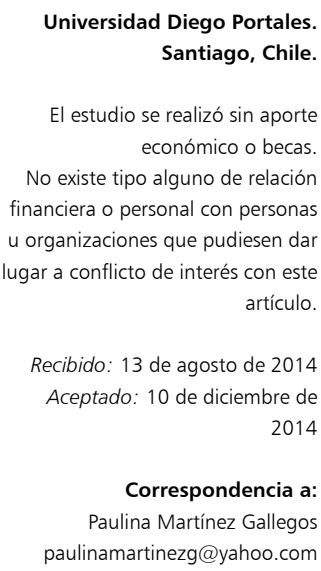
Santiago, Chile.

El estudio se realizó sin aporte económico o becas. No existe tipo alguno de relación financiera o personal con personas u organizaciones que pudiesen dar lugar a conflicto de interés con este

Recibido: 13 de agosto de 2014 Aceptado: 10 de diciembre de

Correspondencia a: Paulina Martínez Gallegos paulinamartinezg@yahoo.com

\author{
Human hydatidosis in children: manifestation of failure in the \\ control and prevention measures. Chile, 2001-2011
}

Background: Hydatidosis is mainly a slowly developing disease, so its appearance in children and adolescents means a relatively recent acquisition; its quantification at this age groups is an indirect mechanism for the evaluation of a prevention and control program. Objective: To characterize human hydatidosis in children aged 0 to 18 years in Chile. Material: Data from the Disease Notification System (2001-2009), hospital discharges (2001-2008), Deaths (2001-2008) and Years of Potential Life Lost (2001-2008) were used. Results: The incidence rate for the period was 4.4 cases per 100.000 inhabitants, male dominated. Hospital discharges rate for the period was 40.3 discharges per 100.000 inhabitants. The mortality rate for the period was 0.13 deaths per 100000 inhabitants. Conclusions: The incidence rates of cases reported, hospital discharges and mortality tend to decrease. However, each new infection reflects the failure of prevention and control, which suggested to incorporate surveillance system tracking and monitoring of cases particularly children's domiciled contacts.

Key words: Hydatidosis; human hydatidosis in children; cases reported; hospital discharges; deaths; PPYLL.

Palabras clave: Hidatidosis; hidatidosis humana en niños; casos notificados; egresos hospitalarios; defunciones; AVPP.

\section{Introducción}

L a hidatidosis es una antropozoonosis causada por estados larvarios del parásito del género Echinococcus granulosus.

Esta enfermedad es de amplia distribución mundial, con zonas de mayor incidencia como la Mediterránea, países del Oriente Medio, África, Australia, Asia y América Latina, destacando en esta última, países como Argentina, Uruguay y Chile.

En nuestro país, esta enfermedad está sujeta al sistema de notificación obligatoria diaria por ser considerada una enfermedad de alto impacto en la salud pública, toda vez que genera costos socio-económicos por concepto de diagnóstico y tratamiento ${ }^{1,2}$. Adicionalmente, esto genera ausentismo escolar e impacto socio-mental no evaluado, tanto del menor de edad como en su familia, como eventuales muertes prematuras.

Considerando que esta enfermedad es mayoritariamente de desarrollo lento y, por lo tanto, infecciones en menores de edad muestran infecciones relativamente recientes e indirectamente es un mecanismo de evaluación del sistema de prevención y control, este trabajo presenta las características generales de la enfermedad en menores entre 0 y 18 años de edad en Chile, según el Sistema de Enfermedades de Notificación Obligatoria (ENO), Egresos Hospitalarios, Defunciones y Años de Vida Potencialmente Perdidos (AVPP).

\section{Características generales}

La hidatidosis es causada por formas larvarias de diferentes géneros del parásito de la Clase Cestoda, Orden Cyclophyllidea, Familia Taeniidae, Género Echinococcus $^{3}$. El ciclo parasitario incluye dos hospederos:

- Hospedero definitivo o carnívoro (particularmente el perro). En él, los parásitos desarrollan la fase adulta o estrobilar en el intestino canino.

- Hospedero intermediario, es el herbívoro u omnívoro (con diferentes especies de importancia epidemiológica, especialmente ovino, caprino, bovino o porcino), donde se desarrollan las formas larvarias o metacéstode, en forma de quiste ("quiste hidatídico") y en diferentes tejidos pero, frecuentemente en hígado y pulmón.

El hombre se ubica dentro de los hospederos intermediarios, siendo un hospedero accidental o paraténico ${ }^{4}$. El hospedero definitivo (perros) elimina huevos del parásito a través de sus fecas, los que contaminan el medio ambiente (suelo, agua, alimentos) pero también quedan en el 
pelaje del animal, siendo de importancia epidemiológica relevante en la transmisión en menores de edad, dada la cercanía que se produce con las mascotas, que los acarician y luego se llevan sus manos a la boca ${ }^{5}$. Si bien el contagio ocurre a cualquier edad, es más fácil en los primeros años de vida por las costumbres de los menores de edad, que contribuye a la ingestión de huevos desde las fecas de los hospederos definitivos ${ }^{6}$.

El estado larval del E. granulosus produce el quiste equinococócico, que tiene una fase de crecimiento variable, donde influyen características tanto del hospedero como del parásito. En los menores de edad, por las características histológicas de sus tejidos, que en general son más laxos, se puede producir un crecimiento del quiste hidatídico a mayor velocidad y tamaño, donde se observan principalmente quistes pulmonares de mayor tamaño ${ }^{7,8}$.

El período de incubación y la presentación clínica es muy variable, fundamentalmente en niños, donde con frecuencia los quistes no inducen síntomas clínicos hasta lograr un tamaño determinado?.

El diagnóstico en menores de edad normalmente se produce una vez que se presenta sintomatología o bien en forma accidental. La confirmación diagnóstica es mediante sus manifestaciones clínicas, imágenes y pruebas serológicas, siendo necesario la asociación entre ellas ${ }^{10}$.

De la situación de equilibrio entre el quiste hidatídico y su hospedero, dependerá el crecimiento, tamaño y consecuentemente, el compromiso de salud, el tratamiento, así como el seguimiento y el tratamiento con antiparasitarios (albendazol) o cirugías (en sus diferentes formas) ${ }^{10,11}$.

\section{Situación epidemiológica}

En Chile, la especie relevante es E. granulosus, de amplia distribución mundial, con países endémicos como Perú, Brasil, Uruguay, Argentina y el nuestro, asociada fundamentalmente a zonas rurales dedicadas a la ganadería, siendo el ovino el hospedero intermediario animal de mayor relevancia epidemiológica. Otras especies animales también pueden ser afectadas, al igual que la ocurrencia de ciclo urbano ${ }^{12}$.

En nuestro país, a pesar del importante cambio en el perfil epidemiológico de la carga de enfermedad, aún persisten enfermedades zoonóticas, como esta antropozoonosis parasitaria. Esto tiene un gran impacto en pérdidas económicas y connotación social, siendo la enfermedad parasitaria de mayor importancia en el país que puede ser absolutamente prevenida. Así, sigue siendo un problema de salud pública no abordado en forma integral y que en consecuencia permanece no resuelto ${ }^{6}$.

Las estadísticas oficiales hablan de una reducción tanto en la incidencia (297 casos en 2001 a 267 casos en 2009); egresos hospitalarios (1.150 egresos en 2001 a 498 egresos en 2008); como defunciones (31 muertes en 2001 a 15 muertes en 2009) ${ }^{6}$; sin embargo, es conocido que esta enfermedad presenta sub-diagnóstico, sub-notificación $\mathrm{y}$ adicionalmente se ha de considerar que toda infección en menores de edad, particularmente entre 0 y 4 años, se traduce en nuevas infecciones y consecuentemente en un fracaso de las medidas de prevención y control.

\section{Métodos}

Estudio descriptivo y retrospectivo, utilizando datos secundarios. Se utilizaron las notificaciones de caso, provenientes del Sistema ENO (Enfermedades de Notificación Obligatoria) del MINSAL, entre 2001-2011. Se seleccionaron los códigos de esta enfermedad de acuerdo a la Clasificación Internacional de Enfermedades (CIE10), siendo los códigos B67.0 (Infección del hígado debida a Echinococcus granulosus); B67.1 (Infección del pulmón debida a Echinococcus granulosus); B67.2 (Infección de hueso debida a Echinococcus granulosus); B67.3 (Infección de otro órgano y de sitios múltiples por Echinococcus granulosus); B67.4 (Infección debida a Echinococcus granulosus, sin otra especificación); B67.5 (Infección del hígado debida a Echinococcus multilocularis); B67.6 (Infección de otros órganos y de sitios múltiples por Echinococcus multilocularis); B67.7 (Infección debida a Echinococcus multilocularis, sin otra especificación); B67.8 (Equinococosis del hígado, no especificada); B67.9 (Equinococosis, otra y la no especificada) $^{13}$.

También se emplearon los registros de los egresos hospitalarios, 2001-2011, con iguales códigos ${ }^{14}$.

Adicionalmente se utilizaron los datos provenientes del registro de mortalidad nacional 2001-2011, con iguales códigos.

Se calcularon los AVPP 2001-2011, por años, sexo y grupos de edad entre 0 y 18 años, basados en la esperanza de vida publicada por el Instituto Nacional de Estadísticas $(\mathrm{INE})^{15}$.

Se calcularon las tasas brutas por año y región, utilizando la población INE a mitad del período, tanto para los casos ENO como egresos hospitalarios y defunciones ${ }^{16}$.

Se realizó un análisis descriptivo, considerando las características de los menores de edad como sexo, edad, diagnóstico y condición de egreso. A la vez, se describió la evolución tanto geográfica -reflejo en ocasiones de desigualdad socioeconómica-, como temporal para las tasas de incidencia ENO, egresos hospitalarios y defunciones.

Para los diferentes análisis se emplearon los programas Excel, SPSS 10.0 y Stata 11.0.

\section{Resultados}

\section{Vigilancia ENO}

Se describe una variación desde 79 casos en 2001 (58,2\% hombres) a 18 en 2011 (50\% cada sexo), con un promedio anual cercano a 66 casos. 


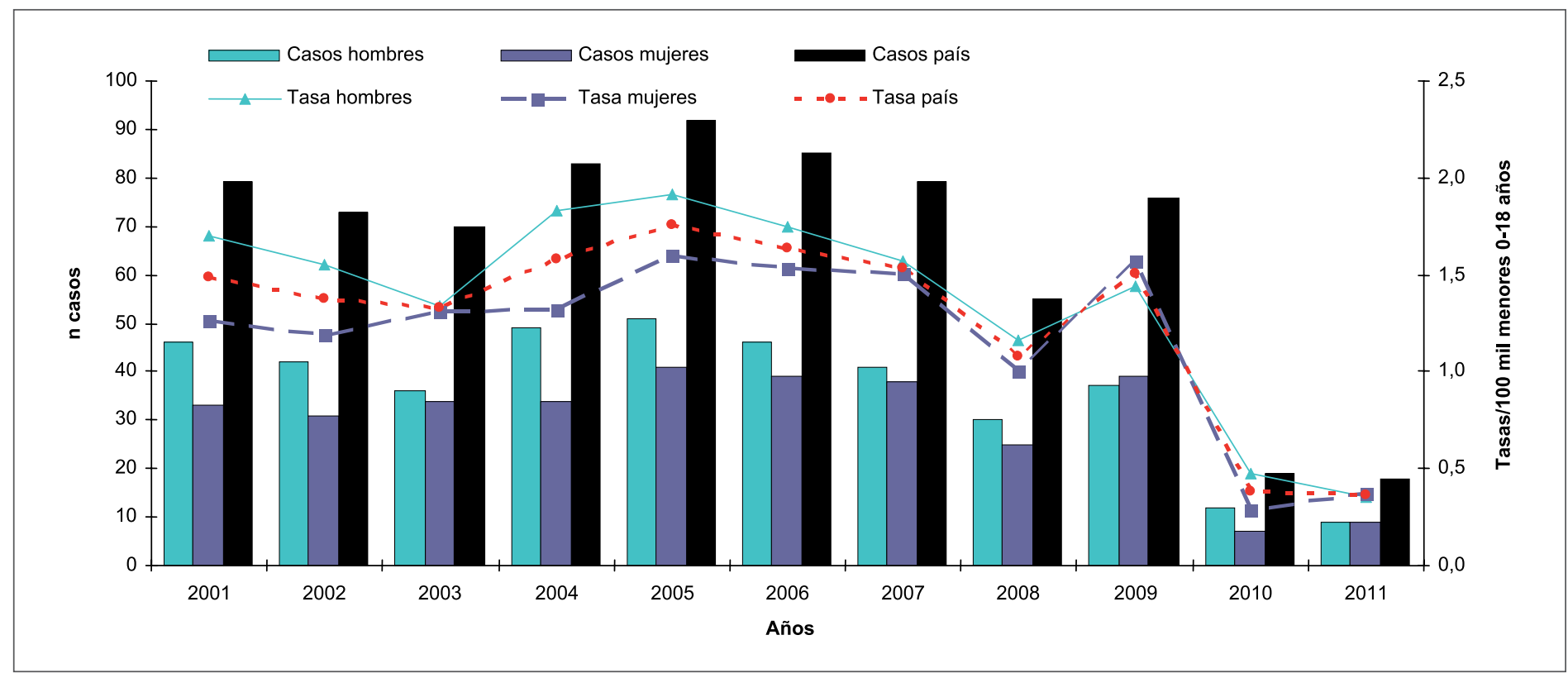

Figura 1. Distribución de casos y tasas de incidencia por hidatidosis (ENO) por sexo. Chile, Menores entre 0 y 18 años, $2001-2011$.

Tabla 1. Casos notificados (ENO) y tasas de hidatidosis humana según año y sexo. Menores entre 0 y 18 años. Chile, 2001-2011

\begin{tabular}{|ccccccc|}
\hline Año & $\begin{array}{c}\text { Casos } \\
\text { hombres }\end{array}$ & $\begin{array}{c}\text { Tasa } \\
\text { hombres }\end{array}$ & $\begin{array}{c}\text { Casos } \\
\text { mujeres }\end{array}$ & $\begin{array}{c}\text { Tasa } \\
\text { mujeres }\end{array}$ & $\begin{array}{c}\text { Casos } \\
\text { país }\end{array}$ & $\begin{array}{c}\text { Tasa } \\
\text { país }\end{array}$ \\
\hline 2001 & 46 & 0,60 & 33 & 0,42 & 79 & 0,51 \\
2002 & 42 & 0,54 & 31 & 0,39 & 73 & 0,46 \\
2003 & 36 & 0,46 & 34 & 0,42 & 70 & 0,44 \\
2004 & 49 & 0,62 & 34 & 0,42 & 83 & 0,52 \\
2005 & 51 & 0,63 & 41 & 0,50 & 92 & 0,57 \\
2006 & 46 & 0,57 & 39 & 0,47 & 85 & 0,52 \\
\hline 2007 & 41 & 0,50 & 38 & 0,45 & 79 & 0,48 \\
2008 & 30 & 0,36 & 25 & 0,30 & 55 & 0,33 \\
2009 & 37 & 0,44 & 39 & 0,46 & 76 & 0,45 \\
\hline 2010 & 12 & 0,14 & 7 & 0,08 & 19 & 0,11 \\
\hline 2011 & 9 & 0,11 & 9 & 0,10 & 18 & 0,10 \\
\hline
\end{tabular}

Tabla 2. Casos notificados (ENO) y egresos hospitalarios de hidatidosis humana según sexo en menores entre 0 y 18 años. Chile, 2001-2011

\begin{tabular}{|lcccccc} 
Sexo & $\begin{array}{c}\mathbf{n} \text { casos } \\
\text { notificados (ENO) } \\
\mathbf{2 0 0 1 - 2 0 1 1}\end{array}$ & $\%$ & $\begin{array}{c}\text { Tasa } \mathbf{x} 100 \\
\text { mil habts. }\end{array}$ & $\begin{array}{c}\text { n egresos } \\
\text { hospitalarios } \\
\mathbf{2 0 0 1 - 2 0 1 1}\end{array}$ & \% & $\begin{array}{c}\text { Tasa x 100 mil } \\
\text { habts. }\end{array}$ \\
Hombre & 399 & 54,7 & 4,9 & 1.183 & 56,6 & 44,8 \\
Mujer & 330 & 45,3 & 4,0 & 906 & 43,4 & 35,6 \\
Total & 729 & 100,0 & 4,4 & 2.089 & 100,0 & 40,3 \\
\hline
\end{tabular}

La tasa de notificación ha cambiado desde 1,48 casos por 100 mil habts., en 2001 a 0,36 casos por 100 mil habts., en 2011 (Figura 1), reflejando -según estos datos- una tendencia a la disminución.

La Tabla 1 muestra el detalle del número de casos y tasas de incidencia de los menores afectados, por año y de acuerdo al sexo, donde se observa que en general las tasas han sido mayores en hombres pero que éstas tienden a igualarse.

La Tabla 2 muestra los casos acumulados y su tasa de incidencia acumulada. La Tabla 3 detalla el número de casos, distribución porcentual y tasa de incidencia según grupo de edad. Considerando el total de casos y de acuerdo al grupo de edad, la mayor concentración porcentual se observa en el estrato de 10-14 años con 35,7\% (260 casos) y una tasa de 17,8 casos por 100 mil habts., entre 10 y 14 años. Sin embargo, el segmento etario que presenta la mayor tasa de notificación corresponde al grupo entre 5 y 9 años, con una tasa de 18,6 casos por 100 mil habts., entre 5 y 9 años (244 casos) y representando $33,5 \%$ del total.

De acuerdo al diagnóstico CIE10, 39,6\% de los casos notificados son clasificados como B67.1 (Infección del pulmón debida a Echinococcus granulosus) seguida con $24,8 \%$ por el código B67.0 (Infección del hígado debida a Echinococcus granulosus).

En relación a la distribución geográfica, las mayores tasas de incidencia se registran en Aysén con 387,4 casos por 100 mil habts., entre 0 y 18 años. Sin embargo, destaca la Región del Bío Bío con el mayor número absoluto de casos notificados (n: 188) (Figura 2). 


\section{Egresos hospitalarios}

El total de egresos para el período en estudio fue de 2.089 (56,6\% hombres). Los egresos se han modificado desde 236 en 2001 (58,1\% hombres) a 138 en 2011 (52,2\% hombres), con un promedio anual cercano a 190 egresos. El mayor número de egresos se registró en 2003 (n: 265).

La tasa de egresos hospitalarios se ha trasladado desde 4,4 a 2,8 egresos por 100 mil habts., entre 0 y 18 años, registrando la mayor tasa el 2003 con 5 egresos por 100 mil habts., entre 0 y 18 años. En este período de estudio se observó una tendencia al decrecimiento (Figura 3).

Las Tablas 2 y 3 exponen las características de los menores egresados por esta causa y su distribución por sexo y grupos de edad.

El grupo de edad de mayor concentración de egresos hospitalarios fue 10 a 14 años (765 egresos); seguido por el segmento de 5 a 9 años (647 egresos).

De acuerdo al tiempo de estadía, se registró una mediana de 8 días (1-120 días); hombres 8 días (1-120 días) y mujeres 8 días (1-85 días).

Según diagnóstico CIE10, 50\% fueron clasificados como B67.9 (Equinococosis, otra y la no especificada), seguido por B67.8 (Equinococosis del hígado, no especificada) con $29,9 \%$.

Según la condición de egreso, 95,5\% egresaron vivos y sólo 4,5\% fallecidos, siendo similar en ambos sexos.

El análisis por sistema de previsión muestra que $94,7 \%$ de los menores egresados eran beneficiarios del Fondo Nacional de Salud (FONASA).

La observación de las tasas de incidencia regional, refleja que la mayor es en Aysén con 532 egresos por 100 mil habts., entre 0 y 18 años. Sin embargo, se ha destacar que la región de La Araucanía registra el mayor número absoluto de egresos hospitalarios (n: 626) (Figura 4).

\section{Mortalidad}

En el período de estudio, las defunciones alcanzan a siete pacientes (mujeres $71,4 \%$ ), registrándose sólo en los años 2001, 2003, 2007 y 2008 (3, 1, 2 y 1 muerte, respectivamente).

La tasa de mortalidad evidencia una tendencia al decrecimiento (Figura 5).

La edad mediana al fallecer fue 14 años (3-18 años); hombres 14,5 años (12-17 años); mujeres 14 años (3-18 años).

El análisis del diagnóstico CIE10, registra que 57,1\% son clasificados como B67.8 (Equinococosis del hígado, no especificada) y 42,9\% como B67.9 (Equinococosis, otra y la no especificada).

Tabla 3. Casos notificados (ENO) y egresos hospitalarios de hidatidosis humana según grupos de edad. Menores entre 0 y 18 años. Chile, 2001-2011

\begin{tabular}{|ccccccc|}
$\begin{array}{c}\text { Edad } \\
\text { (años) }\end{array}$ & $\begin{array}{c}\mathbf{n} \text { casos } \\
\text { notificados } \\
\text { (ENO) } \mathbf{2 0 0 1 - 2 0 1 1}\end{array}$ & $\%$ & $\begin{array}{c}\text { Tasa } \mathbf{x} \mathbf{1 0 0} \\
\text { mil habs. }\end{array}$ & $\begin{array}{c}\text { n egresos } \\
\text { Hospitalarios } \\
\mathbf{2 0 0 1 - 2 0 1 1}\end{array}$ & \% & $\begin{array}{c}\text { Tasa x 100 mil } \\
\text { habs. }\end{array}$ \\
\hline $0-4$ & 67 & 9,2 & 5,4 & 200 & 9,6 & 16,1 \\
\hline $5-9$ & 244 & 33,5 & 18,6 & 647 & 31,0 & 49,4 \\
\hline $10-14$ & 260 & 35,7 & 17,8 & 765 & 36,6 & 52,5 \\
\hline $15-18$ & 158 & 21,7 & 13,4 & 477 & 22,8 & 40,4 \\
\hline Total & 729 & 100,0 & 4,4 & 2.089 & 100,0 & 40,3 \\
\hline
\end{tabular}

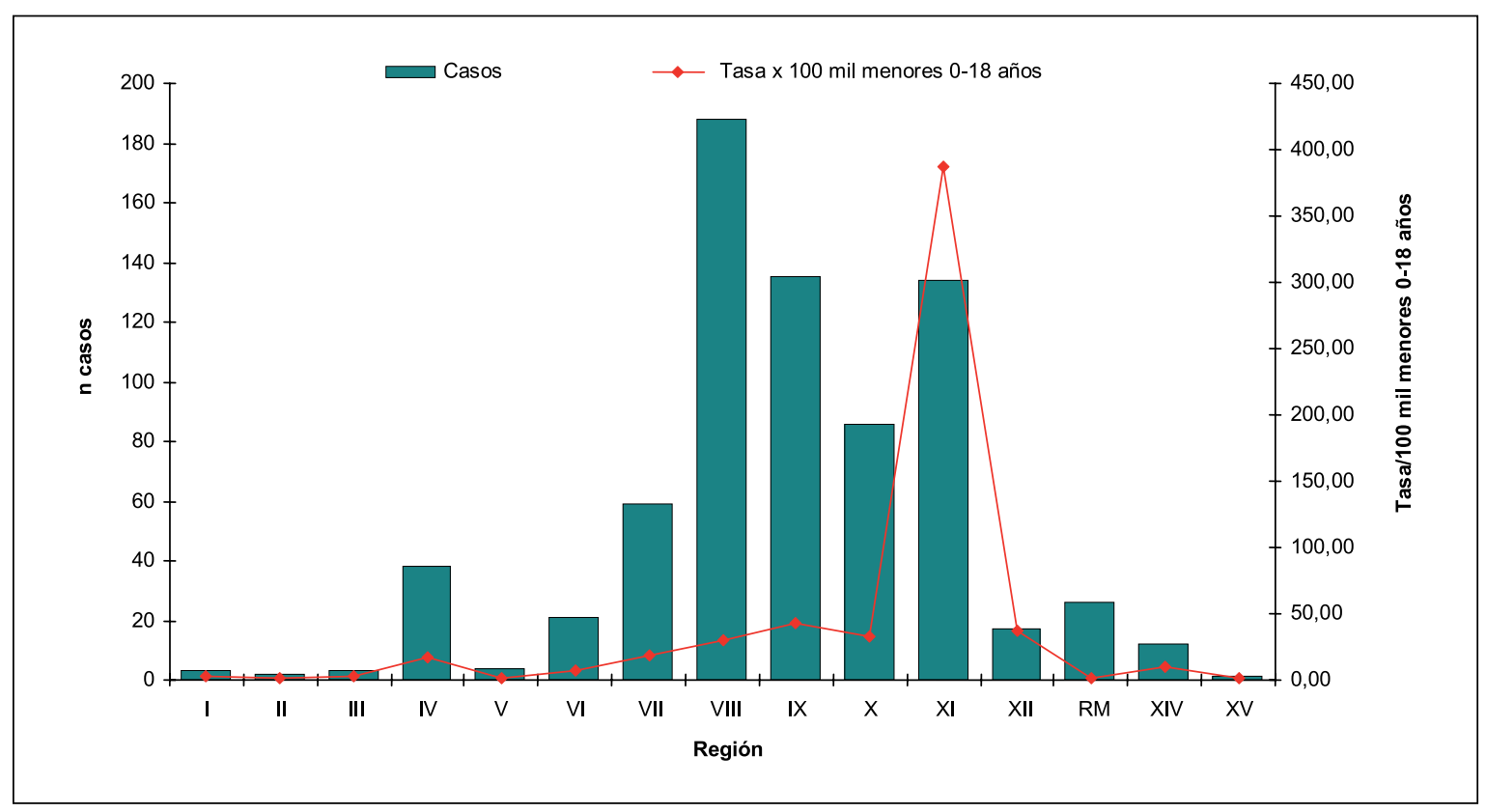

Rev Chilena Infectol 2015; 32 (2): 158-166
Figura 2. Distribución geográfica de casos y tasas de incidencia por hidatidosis (ENO). Chile, Menores entre 0 y 18 años, 2001-2011. 
La evaluación geográfica mediante las tasas de mortalidad regional muestra que la Región de Coquimbo es la mayor, con 1,4 defunciones por 100 mil habts., entre 0-18 años, seguido por La Araucanía con 0,6 muertes por 100 mil menores entre 0 y 18 años (Figura 6).

De acuerdo al nivel de instrucción de los fallecidos, $42,9 \%$ registra un nivel escolar básico -llama la atención en este grupo un adolescente de 18 años-, mientras igual porcentaje $(28,6 \%)$ se observa tanto para escolaridad media como para sin escolaridad; dentro de esta última categoría destaca un joven de 14 años.

Al comparar el número de defunciones y tasas de mortalidad, del registro nacional de mortalidad y según condición en los egresos hospitalarios, se observa, en ambas, una disminución; destaca, sin embargo, la no concordancia entre los datos (Tabla 4).

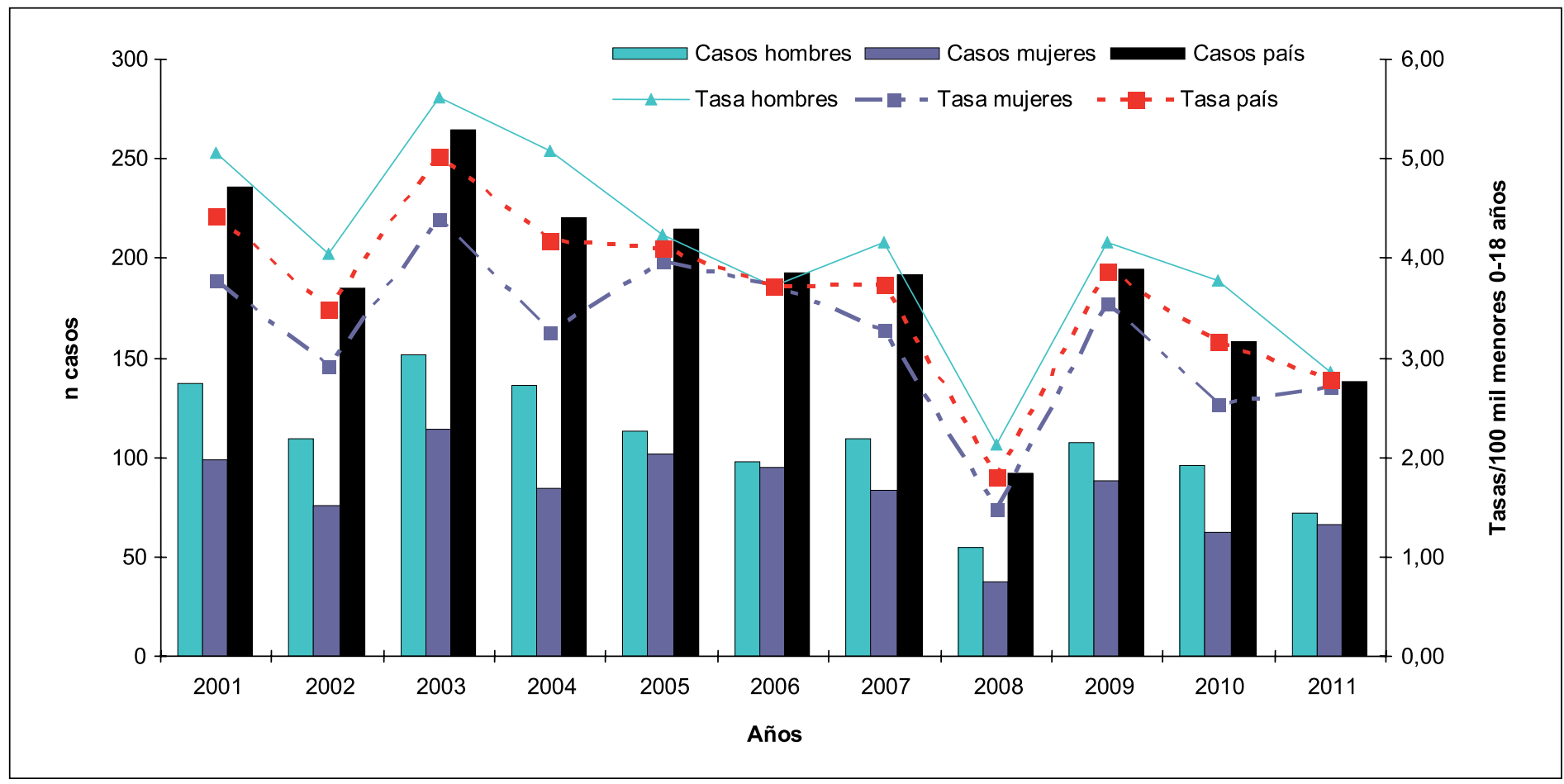

Figura 3. Casos y tasas de egresos hospitalarios por hidatidosis. Chile, Menores entre 0 y 18 años, 2001-2011.

Figura 4. Distribución geográfica de números y tasas de egresos hospitalarios por hidatidosis. Chile, Menores entre 0 y 18 años, 2001-2011.

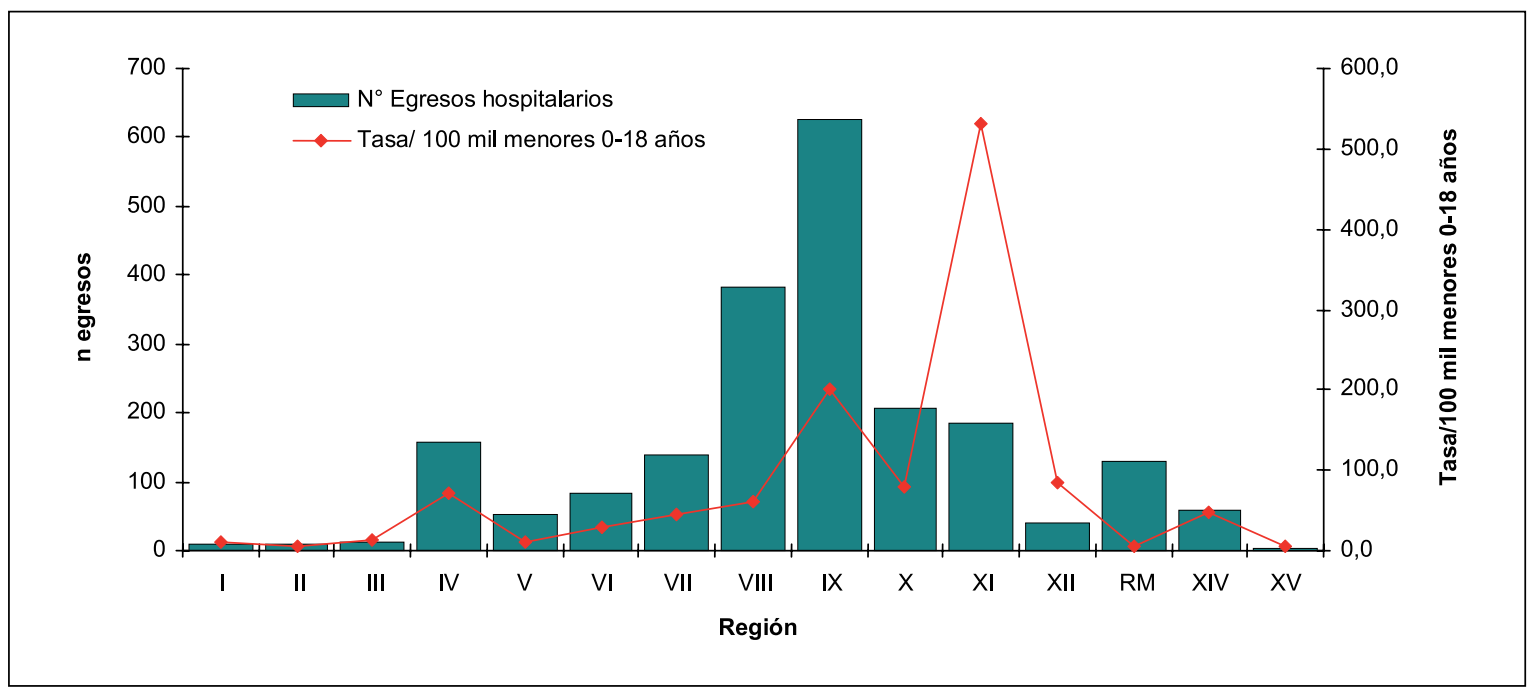




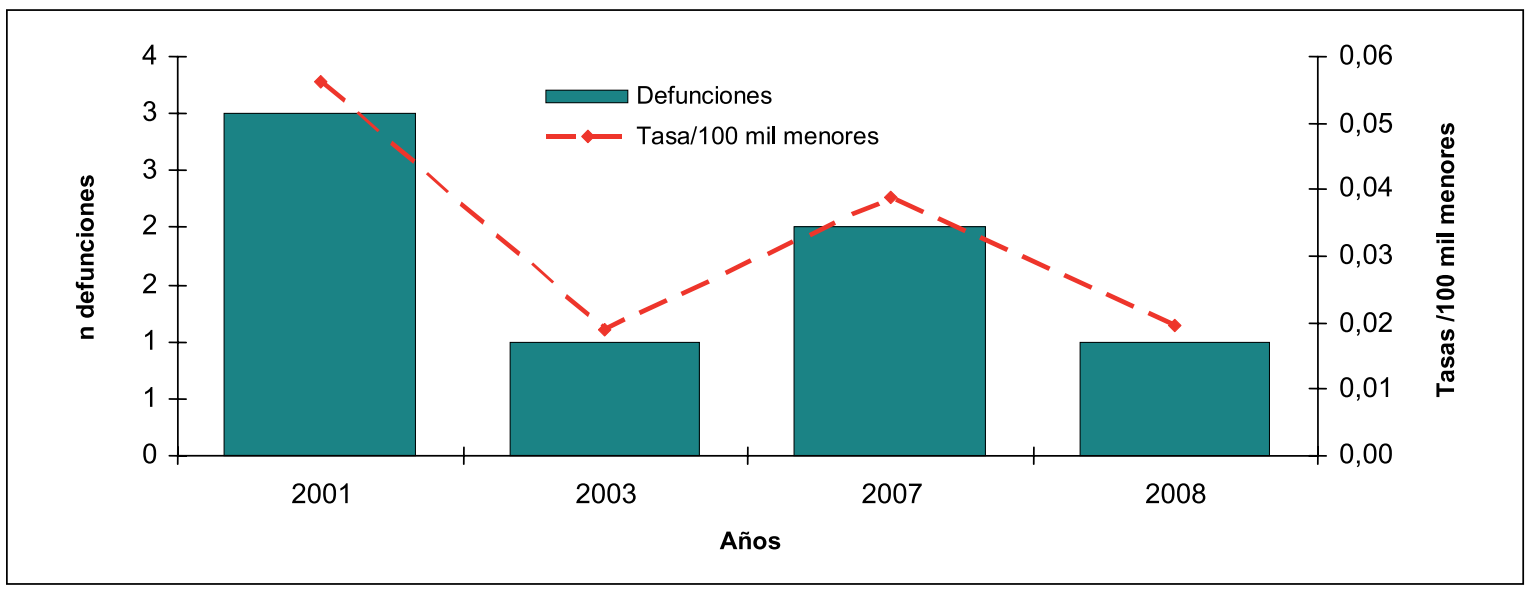

Figura 5. Número y tasas de defunciones por hidatidosis. Chile, menores entre 0 y 18 años, 2001-2011.
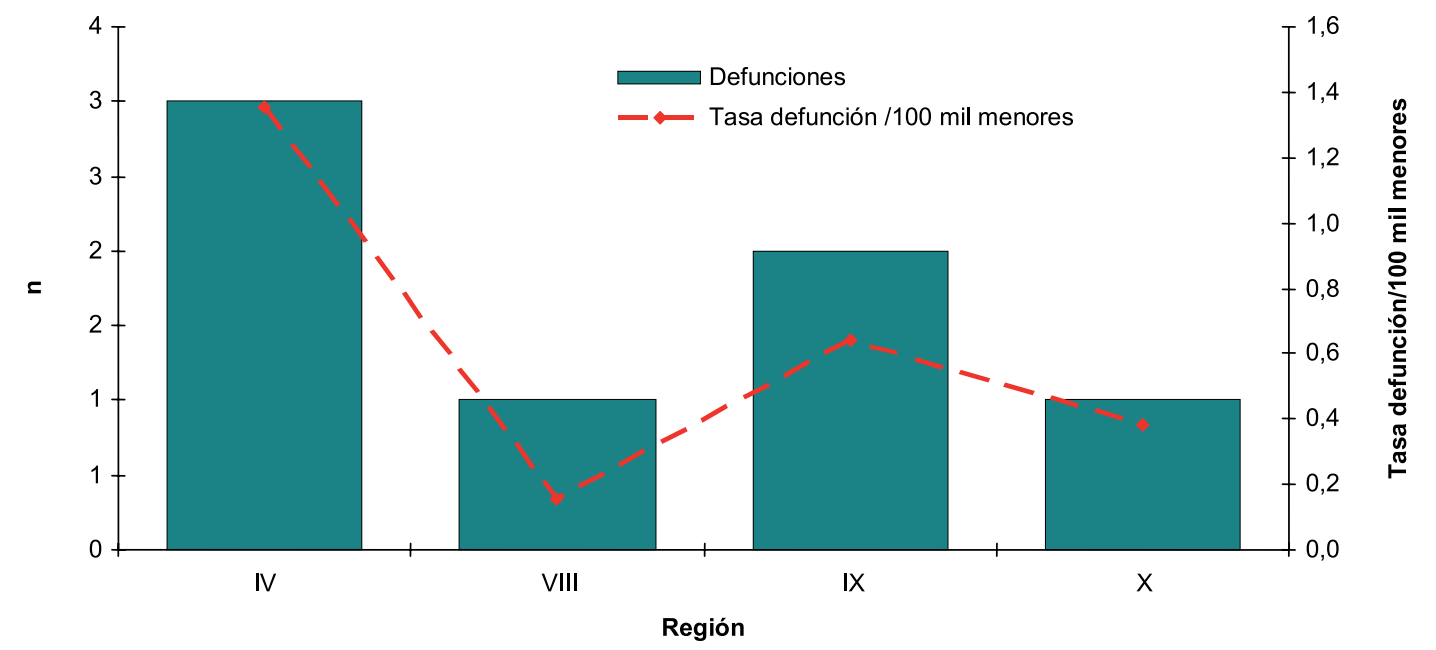

Tabla 4. Defunciones y tasa de mortalidad de hidatidosis humana, según Registro Nacional y condición de egresos hospitalarios, menores entre 0 y 18 años. Chile, 2001-2011

\begin{tabular}{|c|c|c|c|c|c|}
\hline Años & $\begin{array}{c}\text { n defunciones } \\
\text { Registro Nacional }\end{array}$ & $\begin{array}{c}\text { Tasa por } \\
100.000 \text { habts. }\end{array}$ & $\begin{array}{l}\text { n defunciones } \\
\text { egresos hospitalarios }\end{array}$ & $\begin{array}{c}\text { Tasa por } \\
100.000 \text { habts. }\end{array}$ & $\begin{array}{c}\% \text { muertes en egresos } \\
\text { hospitalarios }\end{array}$ \\
\hline 2001 & 3 & 0,06 & 54 & 1,01 & 22,9 \\
\hline 2002 & 0 & 0 & 38 & 0,72 & 20,5 \\
\hline 2003 & 1 & 0,02 & 0 & 0 & 0 \\
\hline 2004 & 0 & 0 & 0 & 0 & 0 \\
\hline 2005 & 0 & 0 & 0 & 0 & 0 \\
\hline 2006 & 0 & 0 & 0 & 0 & 0 \\
\hline 2007 & 2 & 0,04 & 0 & 0 & 0 \\
\hline 2008 & 1 & 0,02 & 1 & 0,02 & 1,1 \\
\hline 2009 & 0 & 0 & 0 & 0 & 0 \\
\hline 2010 & 0 & 0 & 0 & 0 & 0 \\
\hline 2011 & 0 & 0 & 0 & 0 & 0 \\
\hline Total & 7 & 0,13 & 93 & 1,79 & 4,5 \\
\hline
\end{tabular}




\section{Años de vida potencial perdidos}

En el período estudiado se registra una pérdida de 458,81 años de vida por el fallecimiento prematuro de siete menores de edad, correspondiendo al impacto que produjo esta enfermedad sobre los AVPP por muerte prematura y por lo tanto, en años de productividad para el país.

Los AVPP han disminuido 67,9\% entre el 2001 (AVPP: 207,22) y 2008 (AVPP: 66,45). Las mujeres $(345,73$ AVPP por cinco defunciones) perdieron más AVPP que los hombres (122,48 por dos defunciones).

Del total de grupos etarios el orden de afección es: 10-14 años y 15-18 años (42,9\%); y 0-4 años (14,3\%). En hombres: $10-14$ años y $15-18$ años (50\% respectivamente). En mujeres: $10-14$ años y $15-18$ años (40\%); y $0-4$ años $(20 \%)$.

\section{Discusión}

En el período de estudio y según los casos ENO y egresos hospitalarios, los hombres fueron los más afectados ( 58,2 y $56,6 \%$, respectivamente).

De acuerdo al último registro de casos ENO, del total de casos notificados (todas las edades) $21,4 \%$ corresponde a menores entre 0 y 18 años. Mientras que para el registro de egresos hospitalarios, para el período 2001-2011 y también considerando todas las edades, $19,8 \%$ se concentra en los menores entre 0 y 18 años de edad.

Recordemos que esta enfermedad es considerada una afección crónica, siendo más fácil de adquirir en los primeros años de vida y según Félix Dévé "es la enfermedad de las manos y las rodillas en el suelo, los pantalones cortos y los juegos con perros, donde los niños por sus hábitos se comportan de manera similar a los herbivoros, donde aproximan su boca al suelo y así a las oncósferas". Sin embargo, -dado el tipo de desarrollo-, afecta particularmente a edades productivas, impactando considerablemente sobre la economía nacional ${ }^{17}$.

Al igual que en el análisis país, en este grupo se observa discrepancia entre los casos ENO (n: 729) y los egresos hospitalarios (n: 2.089), que reafirma lo mencionado por Rodríguez (2013). Se recomienda corregir los casos notificados de acuerdo a los egresos hospitalarios descontando las re-hospitalizaciones -estimadas en 10\%-, obteniéndose de esta manera una aproximación a los casos incidentes acumulados, que en nuestro estudio y según esta corrección sería de 1.880 casos versus los 729 registrados ${ }^{18}$.

De acuerdo al diagnóstico según CIE10, en los menores notificados por ENO el mayor diagnóstico corresponde a B67.1 (Infección del pulmón debida a Echinococcus granulosus) (39,6\%), lo que es coincidente con la literatura científica ${ }^{19,8}$, que describe mayor frecuencia de presentación de casos pulmonares en menores de edad. Sin embargo, esta tendencia no es observada en los egresos hospitalarios, donde $50 \%$ son clasificados como B67.9 (Equinococosis, otra y la no especificada), seguido por B67.8 (Equinococosis del hígado, no especificada) con $29,9 \%$

De acuerdo a la distribución geográfica, la región que registra mayor tasa de notificación ENO y egresos hospitalarios es Aysén. Sin embargo, destaca la Región de La Araucanía con el mayor número absoluto tanto de casos ENO como egresos hospitalarios. Se ha de recordar que la Región de La Araucanía presenta el PIB per cápita más bajo dentro del país, con los niveles más elevados de extrema pobreza y pobreza ${ }^{6}$.

Al comparar el tiempo de estadía con el registro nacional (todas las edades) no se observa diferencia significativa, encontrándose en los menores una estadía mediana de 8 días y en la población total una mediana de 9 días, lo que reafirma el avance médico/tecnológico que permite resoluciones en menor tiempo y consecuentemente menor costo tanto económico como social.

De acuerdo al análisis de la mortalidad, se aprecia para el período un bajo número de muertes por esta causa e incluso, no se registran defunciones desde el año 2008, lo que puede ser explicado por la eficiencia en los tratamientos una vez pesquisados los casos y la prioridad de atención a menores de edad.

En este período se registran más defunciones en mujeres (71,4\%; n: 5); sin embargo, se ha de destacar el bajo número total (n: 7).

De conformidad al nivel de instrucción de los fallecidos, la mayoría -esperado dadas sus edades-, presenta nivel escolar básico (42,9\%). Llama la atención el registro de un adolescente de 18 años con nivel básico y un joven de 14 años sin escolaridad. Se ha de considerar que en nuestro país es obligatorio 12 años de educación.

Al observar la proporción de muertes en egresos hospitalarios destaca la discrepancia con el registro nacional de defunciones. Esta diferencia probablemente sea debido a las actividades de validación y corrección de datos, entre ellos, consulta a médicos emisores de los certificados de defunción y comparación con otras bases de datos. Esto puede modificar los datos originales y de ser esta la explicación, se hace necesaria una mejora en la calidad de clasificación de muertes por parte de los equipos de salud tratantes, particularmente los médicos -dado que son estos profesionales quienes deben completar el certificado de defunción-, donde a nivel general se ha descrito problemas en la exactitud y precisión de causas de muerte ${ }^{20}$.

Se produjo la pérdida de 458,81 años de vida por el fallecimiento prematuro de sólo siete menores de edad. Este es el impacto que produjo esta enfermedad sobre la mortalidad en AVPP y, consecuentemente, en años de producción para el país. No obstante, el bajo número 
de defunciones, se ha de considerar el fuerte impacto que tiene esta enfermedad en menores de edad no sólo a nivel individual y familiar, sino también a nivel de sociedad, porque las muertes por hidatidosis en gente joven se relacionan a mayor pérdida de años de vida $y$, consecuentemente, mayor pérdida en años de producción. Se ha de considerar que estas defunciones son muertes evitables, dado que es una enfermedad que tiene medidas de control, normas de diagnóstico y tratamiento.

A la vez, se ha de recordar que nuestro país ha ratificado la Convención de los Derechos del Niño; en su Artículo 24 hace referencia a la salud, donde los "Estados Partes reconocen el derecho del niño al disfrute del más alto nivel posible de salud"; Adoptar las medidas adecuadas para reducir la mortalidad infantil y en la niñez; Combatir las enfermedades aplicando la tecnología disponible y considerando los riesgos de contaminación del medio ambiente; Asegurar que todos los sectores de la sociedad, y en particular los padres y los niños, conozcan los principios básicos de la salud, la higiene y el saneamiento ambiental; y Desarrollar la atención sanitaria preventiva, entre otros. De acuerdo a esta declaración, cada vez que un menor de edad enferma o fallece a consecuencia de esta enfermedad absolutamente prevenible, se ha de considerar como impedimento para el ejercicio de sus derechos, especialmente el derecho a la salud y consecuentemente a la vida ${ }^{21}$.

Es necesario recalcar en el equipo médico la importancia de la sospecha precoz, especialmente en las regiones de mayor incidencia. Y estrechamente ligado a lo anterior, es considerar el estudio de los contactos de pacientes de toda edad, pero particularmente de los menores de edad, -incluyendo estudios serológicos y de imágenes-, de manera de ofrecer un diagnóstico y tratamientos precoces. Respecto a la relevancia de lo anterior, Anzieta y cols., encontraron antecedentes epidemiológicos de familiares con previo diagnóstico de hidatidosis en $26,6 \%$ de los pacientes menores de edad ${ }^{22}$.

En relación a la hidatidosis, la información disponible confirma que es una enfermedad prevenible y considerando que se conocen los factores condicionantes y su forma de control, en Chile es factible la reducción, mediante acceso a la educación, información y participación de la población en las medidas de control y prevención en los diferentes grupos de riesgo (registro canino, tratamiento antiparasitario, control de población canina); inspección sanitaria de los animales de abasto y sus carnes (en aplicación en las plantas faenadoras del país) y fortalecimiento de los sistemas de vigilancia epidemiológica ${ }^{23,24}$.

Por lo tanto, de acuerdo a la evidencia científica, se hace necesario la implementación de una política de control nacional de la hidatidosis, continúa y a largo plazo, con enfoque multisectorial, inter-programático, que aborde los factores de riesgo y protección y, con disposición asegurada de recursos, lo cual implica necesariamente reconocer que es deber del Estado proteger la salud y alcanzar una mejor calidad de vida para toda la población, particularmente de los menores de edad. Consecuentemente, se debe fijar una agenda de trabajo intersectorial e integral entre los sectores salud, agricultura, educación, desarrollo social, comunidad, academia y sociedad civil en general. Además, esta política debe integrar a los diferentes actores involucrados desde su generación (diseño, implementación y evaluación), aprovechando las experiencias e infraestructura existente.

Antes de finalizar, recordar que la infección se adquiere fundamentalmente en la niñez y los síntomas y sospecha clínica aparecen generalmente en los adultos. Sin embargo, cada nueva infección refleja el fracaso de las medidas de prevención y control y si es en menores -dada la edad- las podemos proyectar como un fracaso actual.

Finalmente, luego del análisis de estas diferentes fuentes de información y el hallazgo de discrepancias, se sugiere en lo inmediato evaluar el sistema de vigilancia epidemiológica e incorporar el manejo de contactos de pacientes, especialmente el seguimiento de menores de edad que habiten en igual domicilio, considerando que todo caso de un menor de edad significa una infección reciente y consecuentemente un déficit en la salud pública.

\section{Resumen}

Introducción: La hidatidosis es mayoritariamente una afección de desarrollo lento $\mathrm{y}$, por lo tanto, infecciones en la infancia y adolescencia traducen infecciones relativamente recientes; su cuantificación a estas edades es, indirectamente, un mecanismo de evaluación del sistema de prevención y control de la misma. Objetivo: Caracterizar epidemiológicamente la hidatidosis humana en pacientes entre 0 y 18 años de edad en Chile. Material: Se utilizaron datos del Sistema de Notificación Obligatoria (2001-2011), Egresos Hospitalarios (2001-2011), Defunciones (2001-2011) y Años de Vida Potencialmente Perdidos (2001-2011). Resultados: La tasa de incidencia para el período fue 4,4 casos por 100 mil habts., con predominio masculino. La tasa de egresos hospitalarios para el período fue 40,3 egresos por 100 mil habts. La tasa de mortalidad para el período fue de 0,13 defunciones por 100 mil habts. Conclusiones: Las tasas de incidencia de casos notificados, egresos hospitalarios y mortalidad tienden a disminuir. Sin embargo, cada nueva infección refleja el fracaso de las medidas de prevención y control. Se sugiere incorporar al sistema de vigilancia un seguimiento y monitoreo de casos contactos particularmente de menores de edad que compartan domicilio. 


\section{Referencias bibliográficas}

1.- Gobierno de Chile. Ministerio de Salud. Reglamento sobre notificación de enfermedades transmisibles de declaración obligatoria $\mathrm{N}^{\mathrm{o}} 158$. Santiago, 22 de octubre de 2004.

2.- Gobierno de Chile. Ministerio de Salud. Normas Técnicas de Vigilancia de Enfermedades Transmisibles. 2000. http://epi.minsal.cl/epi/ $\mathrm{html} /$ public/enftransmisibles.pdf [accedido el 19 de julio de 2014].

3.- Menezes da Silva A. Human echinococcosis: a neglected disease. Gastroenterol Res Practice 2010; 2010: 583297.

4.- Osorio M, Godoy H. Estudio "Vulnerabilidad Social Frente a Hidatidosis Humana”. Informe Final Versión 2.0, marzo de 2008. Informe no publicado.

5.- Cortés S, Valle C. Hidatidosis humana: Generalidades y situación epidemiológica en Chile según egresos hospitalarios y notificación obligatoria entre los años 2001 y 2005. Rev Chilena Infectol 2010; 27 (4): 329-35.

6.- Martínez P. Caracterización de la mortalidad por hidatidosis humana. Chile, 2000-2010. Rev Chilena Infectol 2014; 31 (1): 7-15.

7.- Rivera M. Hidatidosis en pacientes pediátricos. Realidad local. Complejo Asistencial Víctor Ríos Ruiz. Seminario de Hidatidosis. Los Ángeles. Bío Bío. 26 y 27 de junio de 2014.

8.- Benítes C. Características clínico quirúrgicas y de exámenes auxiliares para hidatidosis en pacientes menores de 15 años del Hospital Nacional Dos de Mayo, durante 1995-2005.
Universidad Ricardo Palma. Facultad de Medicina Humana. Tesis para optar el título profesional de Médico Cirujano. Lima, Perú. 2005.

9.- Frider B, Larrieu E, Odrizola M. Long term outcome of a symptomatic liver hidatidosis. J Hepatol 1999; 30: 228-31.

10.- Noemi I, Viovy A, Zamorano R, Blanco R, Revello D, Vojkovic L, et al. Experiencia clínica. Hidatidosis en la infancia: Albendazol en su tratamiento médico y quirúrgico. Rev Chilena Infectol 2003; 20 (4): 229-34.

11.- Larrieu E. Diagnóstico y tratamiento de la hidatidosis en población escolar: informe preliminar. Arch Argent Pediatr 2002; 100 (6): 448-55.

12.- Huamán I, Marocho L, López T, Gavidia C. Frecuencia de hidatidosis en niños y adolescentes hospitalizados en el Instituto Nacional de Salud del Niño (Período 19962005). Rev Inv Vet Perú 2010; 21 (1): 54-60.

13.- Organización Panamericana de la Salud (OPS). Clasificación Estadística Internacional de Enfermedades y Problemas relacionados con la Salud. Décima revisión. 1995.

14.- Gobierno de Chile. Ministerio de Salud. Datos de Egresos Hospitalarios 2014.

15.- Gobierno de Chile. Ministerio de Salud. Esperanza de vida al nacer (en años) por período y sexo. Chile, 1950-2025. http://deis. minsal.cl/deis/ev/esperanza_de_vida/index.asp [Consultado el 20 de julio de 2014].

16.- Gobierno de Chile. Instituto Nacional de Estadística (INE). Departamento de
Demografía. Programa de proyecciones de la población. Proyecciones de población por grupos de edad y sexo.

17.- Jensen O. Instituto de Zoonosis de Argentina. Comunicación personal. 2014.

18.- Rodríguez B. Situación y evolución temporal de la hidatidosis en Chile 2001-2007. Tesis para optar al grado de Magíster en Salud Pública. Universidad de Chile. Santiago, Chile, 2013.

19.- Retamal C, Pérez C, Noemí I, Aguilera X, Apt W. Evaluación de las técnicas de doble difusión 5 e inmunoelectroforesis en hidatidosis infantil en la casuística del decenio. Rev Chil Pediatr 1994; 65: 251-4.

20.- Antini C. Mortalidad atribuida a insuficiencia cardíaca: calibración de la certificación y codificación de la causa de muerte. Tesis para optar al Grado de Doctor en Salud Pública. Universidad de Chile. Santiago, Chile, 2013.

21.- UNICEF Chile. Texto Oficial de la Convención sobre los Derechos del Niño aprobada por la Asamblea General de las Naciones Unidas el 20 de noviembre de 1989. pp 17.

22.- Anzieta J, Caro M, Fierro C, Rocco E. Quiste hidatídico pulmonar en niños. Cuad Cir 2002; 16: 16-9.

23.- Seremi Región del Bío Bio/Servicio de Salud Bío Bío. Seminario Actualizaciones en Hidatidosis. 26-27 de junio de 2014.

24.- Troncoso C. Algunas zoonosis de bovinos. Planta faenadora de carnes de Temuco, IX Región, Chile 1990-1999. Temuco, Chile. Universidad de la Frontera, 2000. 39 pp. 